\title{
Successful endoscopic surgical treatment of pleuroperitoneal communication in two infant cases
}

\author{
Teizaburo Mori ${ }^{1 *} \mathbb{D}$, Akihiro Fujino ${ }^{1}$, Masataka Takahashi ${ }^{1}$, Ryoya Furugane ${ }^{1}$, Tamotsu Kobayashi ${ }^{1}$, \\ Motohiro Kano ${ }^{1}$, Akihiro Yoneda' ${ }^{1}$, Yutaka Kanamori ${ }^{1}$, Ryutaro Suzuki ${ }^{2}$, Kentaro Nishi ${ }^{2}$, Koichi Kamei ${ }^{2}$ and \\ Masayuki Kitamura ${ }^{3}$
}

\begin{abstract}
Background: Pleuroperitoneal communication (PPC) is an uncommon, but potentially life-threatening complication of peritoneal dialysis (PD). If a fistula does not close with conservative treatment, surgical repair is required. However, approximately half of these patients are forced to shift from PD to hemodialysis. Although it is important to confirm the site of the fistula to achieve a successful surgical treatment, this identification is more difficult in pediatric patients than in adults.

Case presentation: We report two infantile cases of severe PPC associated with PD. In both cases, the age at onset was less than 2 years, and right-sided pleural effusion with dyspnea was observed. PPC was diagnosed by the change in color of the pleural fluid after the injection of a dye into the peritoneal cavity. Peritoneal scintigraphy and singlephoton emission computed tomography and computed tomography (SPECT/CT) were performed, and these were effective in locating the fistula site. Endoscopic surgery (video-assisted thoracic surgery (VATS) and laparoscopic surgery) was performed. Indocyanine green (ICG), which was injected into the abdominal cavity, showed the exact site of the fistula. The fistula was successfully closed by attaching an absorbable sheet to it from the thoracic side and an autograft (the falciform ligament) to it from the abdominal side in one patient. In the other patient, the fistula site was resected and sutured, and reinforced with an absorbable sheet. In both cases, PD was resumed without any complication.
\end{abstract}

Conclusion: We successfully treated two infants of PPC by endoscopic surgery. To identify the fistula site, the ICG navigation method was useful. Even in small infants, PPC can be treated successfully by endoscopic surgical repair if the site of the fistula is identified.

Keywords: Pleuroperitoneal communication, Peritoneal dialysis, Pleural effusion, Video-assisted thoracoscopic surgery, Infant, Indocyanine green

\section{Background}

Pleuroperitoneal communication (PPC) causing massive pleural effusion is an uncommon, but well-known complication among the nephrologists who treat renal

\footnotetext{
*Correspondence: mori-te@ncchd.go.jp

1 Division of Pediatric Surgery, National Center for Child Health and Development, 2-10-1 Okura Setagaya-ku, Tokyo 157-8535, Japan Full list of author information is available at the end of the article
}

insufficiency, and it is potentially life-threatening complication of peritoneal dialysis (PD) [1-3]. Although pleural effusion occurs shortly after PD induction in most cases, it can develop at any time during PD treatment [4]. Pleural effusion in PPC is seen mostly in the right-sided thoracic space [1] and caused by the leakage of peritoneal fluid into the pleural cavity via pleuroperitoneal fistulas [3]. Currently there is no definitive way to accurately 
identify the fistula [5]. In the past reports, approximately half of these patients are forced to shift to hemodialysis $[1,6]$ due to the failure to close the fistula.

In pediatric cases with end-stage renal disease, $\mathrm{PD}$ is a well-established, preferred and safety method to maintain dialysis therapy [1, 7]. Therefore, it is very important to obtain early diagnosis and grapple with proper treatment for PPC. If the fistula does not close with temporary cessation of PD, a surgical approach is required. However, surgical repair often fails because the site of the fistula is usually very difficult to identify in pediatric cases.

In this report, we present two infants with PPC, for whom surgical treatment was successfully performed by identifying the fistula site using currently available methods. To the best of our knowledge, Case 1 is the first reported case where the fistula site was identified from the abdominal side, and Case 2 is the youngest and the smallest patient with PPC in literature.

\section{Case presentation}

\section{Case 1}

A 1 year and 10-month-old girl with Denys-Drash syndrome, weighing $10 \mathrm{~kg}$, was referred to our institute with subacute onset of respiratory distress due to the right-sided pleural effusion (Fig. 1a). She was on PD from 5 months of age. She presented with complaints of dyspnea, following decrease in PD fluid drainage for a month. Artificial ventilation support was started due to severe respiratory failure and a chest tube was placed to drainage the right-sided pleural effusion. When indigo carmine dye $(20 \mathrm{mg} / \mathrm{L})$ was infused into the abdominal cavity through the PD catheter, the dye color was not detected in the drained pleural fluid under the mechanical ventilation. However, when a re-trial was performed under spontaneous respiration after extubation, bluecolored discharge was found in the chest tube, confirming the diagnosis of PPC. PD was discontinued, and hemodialysis was initiated as a conservative treatment for PPC. Since PPC did not resolve after cessation of PD for 3 months, surgical treatment was performed.

Before the surgery, scintigraphy and single-photon emission computed tomography and computed tomography (SPECT/CT) were performed. Ten minutes after $99 \mathrm{~m}$-Tc-Sn-colloid injection into the peritoneal cavity with dialysate, radioactivity was detected in the right pleural cavity. A hot spot remained in the medial area of the central tendon of the right diaphragm and mediastinal angle until the next day (Fig. 1b).

For definitive treatment, thoracoscopic surgery under separate lung ventilation was performed, with the patient placed in a left-sided semi-lateral decubitus position. A dialysate containing indigo carmine $(20 \mathrm{mg} / \mathrm{L})$ and indocyanine green (ICG) $(0.5 \mathrm{mg} / \mathrm{kg} / \mathrm{L})$ was injected into the abdominal cavity to identify the leakage site. Though the pleural fluid was not stained blue, an infrared camera detected fluorescence signal in the pleural effusion (Fig. 2a, b). A cystic lesion with thinned out pleura was noted at the site where the hot spot was observed by scintigraphy, and dialysate accumulated under that site (Fig. 2b, c). After scratching the pleural surface, a sheet of

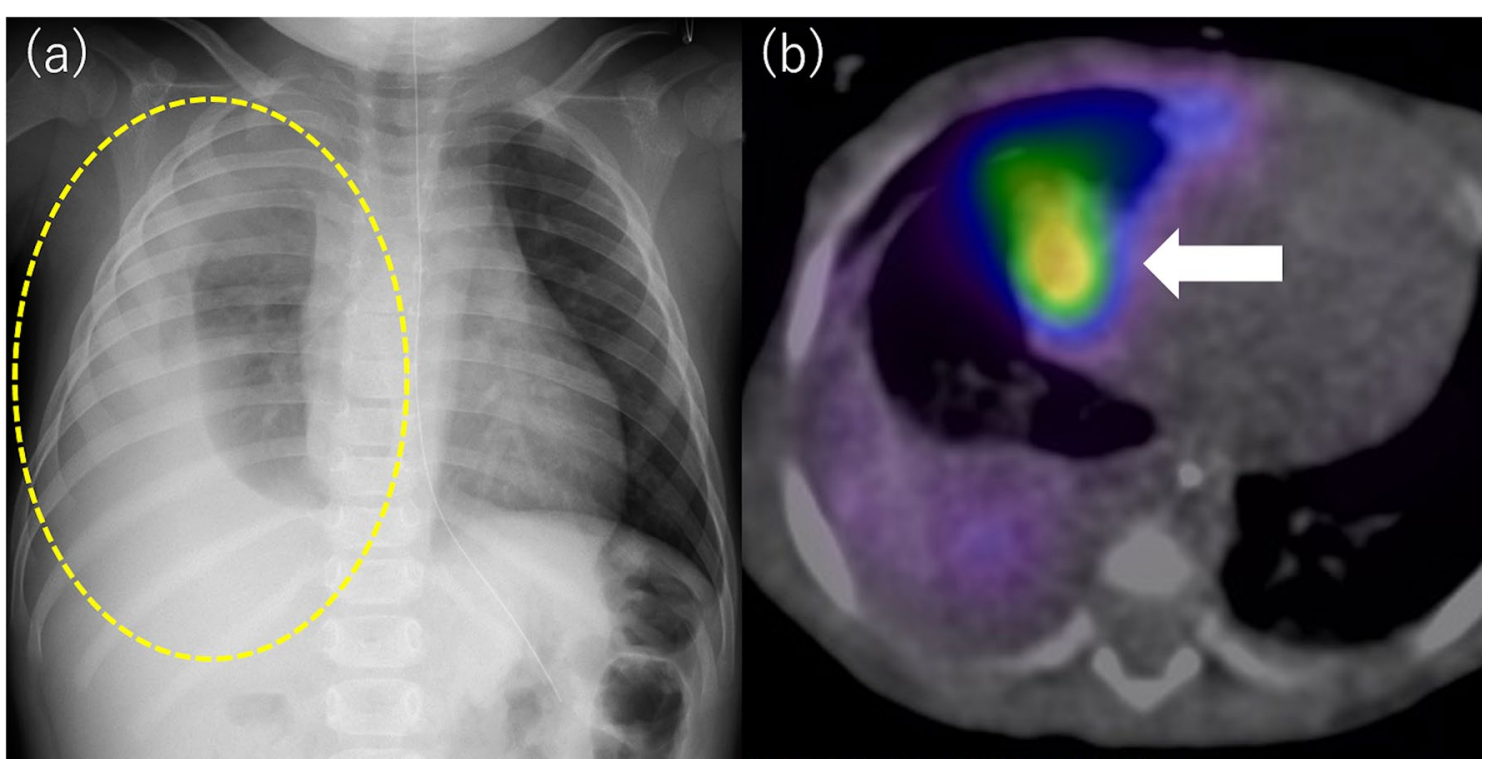

Fig. 1 a A chest radiograph showed a right-sided hydrothorax. b Preoperative scintigraphy demonstrating the radioactive dialysate in the thoracic cavity confirming a PPC. The arrow indicates hot spot which remained for a next day 


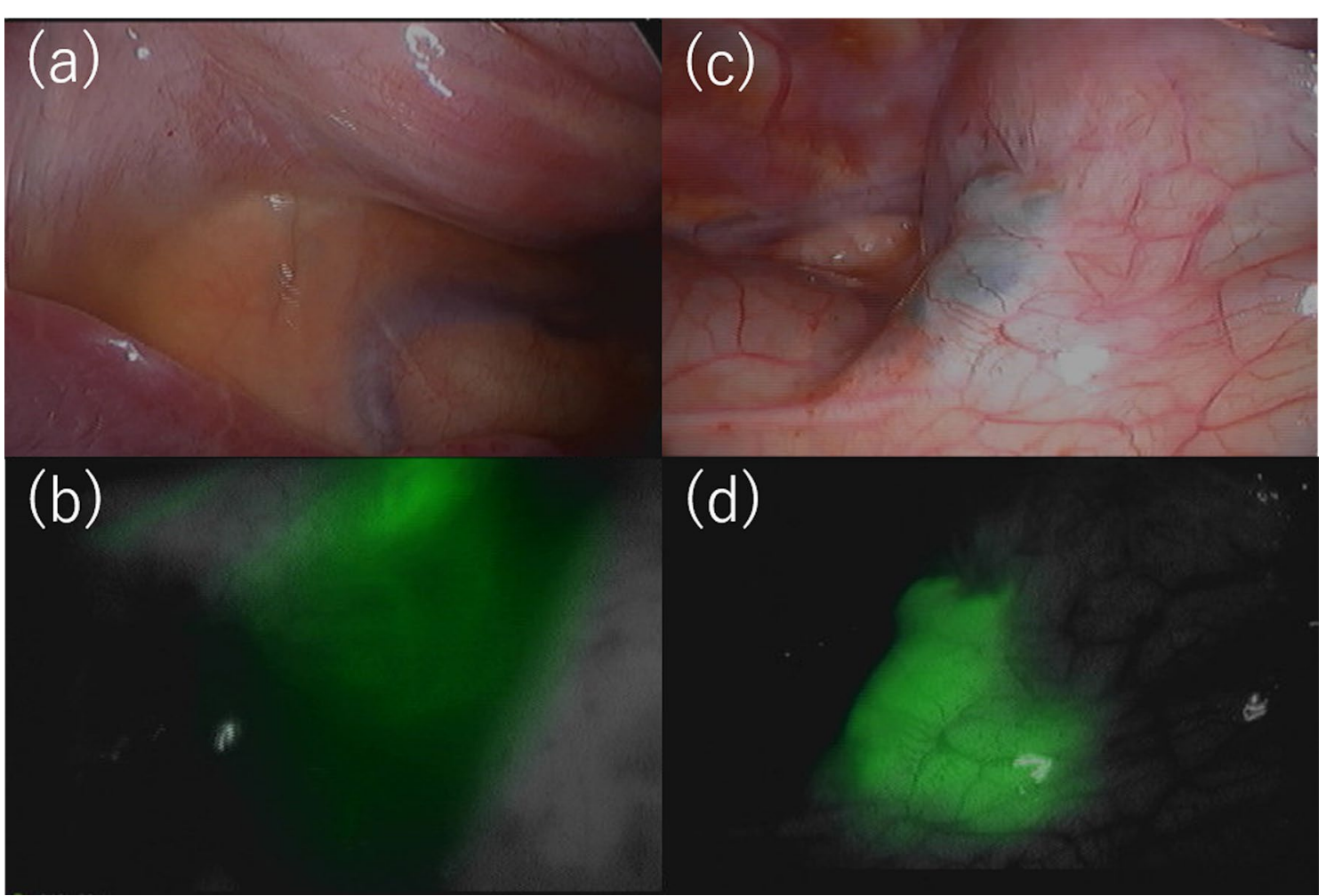

Fig. 2 Thoracoscopic findings. a The pleural fluid was not stained, but an infrared camera detected fluorescence in the pleural effusion (b). A cystic lesion was observed in which the pleura was thinned and dialysate was accumulated under the pleura at the central tendon of the diaphragm of the mediastinal angle $(\mathbf{c}, \mathbf{d})$

absorbable polyglycolic acid felt (Neoveil; Gunze Medical; Tokyo, Japan) was attached to the lesion (Fig. 3a). Following thoracoscopic surgery, laparoscopic PD catheter replacement was performed, and a small hole was found on the peritoneal side of the hot spot. It was thought that the dialysate had likely entered the diaphragm through this hole, after scratching the peritoneal surface, a falciform ligament flap patch was sutured on the hole (Fig. 3b). The patient's postoperative course was uneventful. The patient resumed PD 2 weeks after surgery, and no recurrence of PPC was observed for 10 months.

\section{Case 2}

A 5-month-old boy with Potter syndrome, weighing $6 \mathrm{~kg}$, was admitted to our institute with subacute respiratory distress due to right-sided pleural effusion (Fig. 4a). He was on PD from 2 weeks of age. Artificial ventilation support was started, as the pleural effusion worsened after admission, and a chest tube was placed. Indigo carmine dye $(20 \mathrm{mg} / \mathrm{L})$ was infused through the PD catheter after positive pressure ventilation support was completed, and blue-colored discharge was found in the chest tube after $5 \mathrm{~min}$, confirming the diagnosis of PPC. PD was discontinued, and hemodialysis was initiated as a conservative therapy. Scintigraphy and SPECT/CT detected leakage of the dialysate into the right pleural cavity, but no remarkable hot spots were observed (Fig. 4b). Since PPC did not resolve spontaneously after 3 months of withholding PD, surgery was attempted.

Dialysate containing indigo carmine $(20 \mathrm{mg} / \mathrm{L})$ and ICG $(0.5 \mathrm{mg} / \mathrm{kg} / \mathrm{L})$ was injected into the abdominal cavity, and the entire thoracic cavity was inspected carefully by thoracoscopy under separate lung ventilation. Although the pleural fluid was not stained, an infrared camera detected leakage of fluorescent fluid in the lateral edge of the central tendon of the right diaphragm, where small holes were present (Fig. 5a). The diaphragm lesion including the small holes was excised and sutured directly via a small thoracotomy. A sheet of absorbable polyglycolic acid felt (Neoveil; Gunze Medical; Tokyo, Japan) was fixed with fibrin glue onto the sutured lesion (Fig. 5b). The patient's postoperative course was uneventful. The patient resumed PD 3 weeks later, and no recurrence of PPC was observed during follow-up for 6 months. 


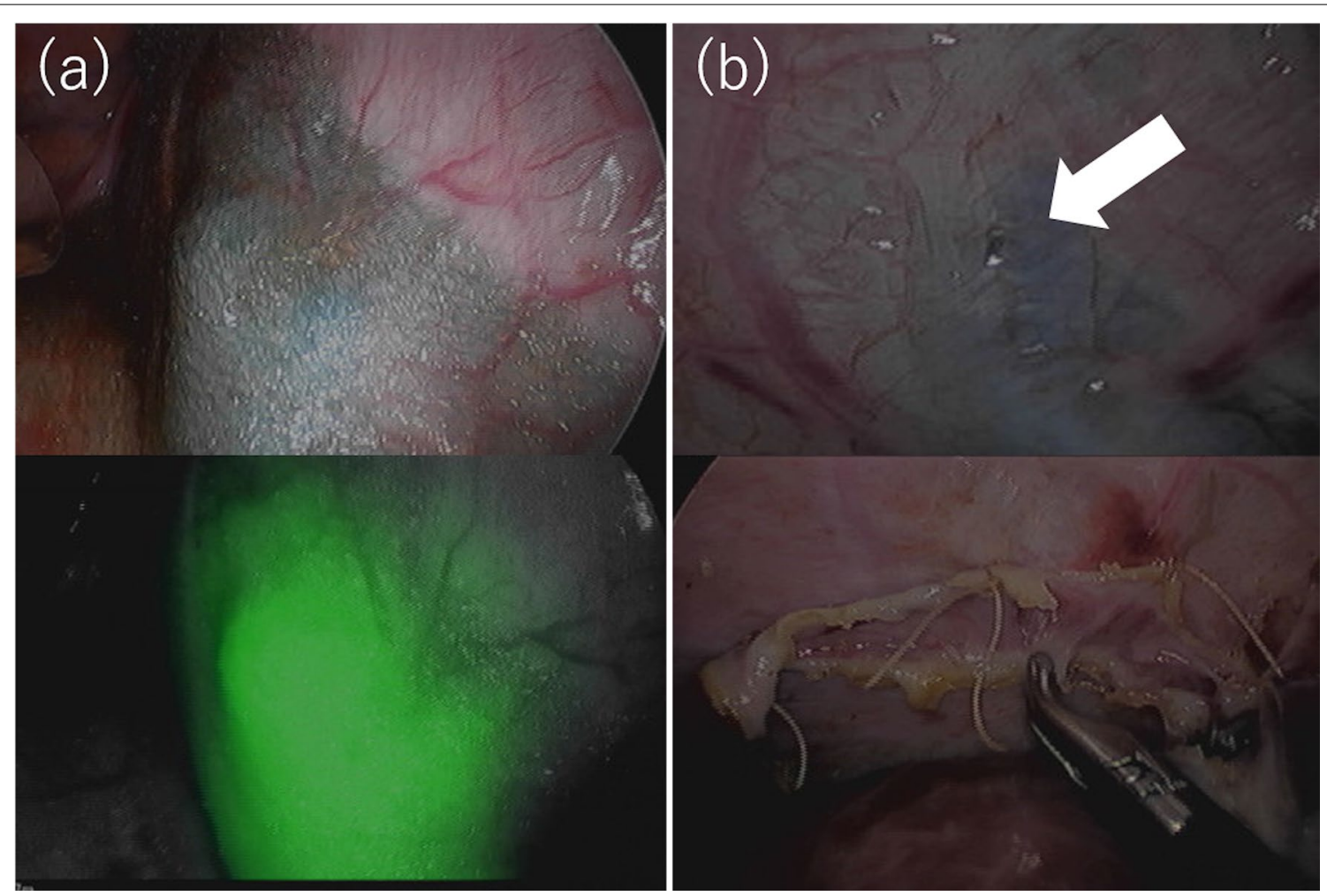

Fig. 3 After treatment findings. a After scratching the pleural surface, a sheet of absorbable polyglycolic acid felt was attached onto the cystic lesion. b The hole (arrow) was covered by suturing a falciform ligament flap patch

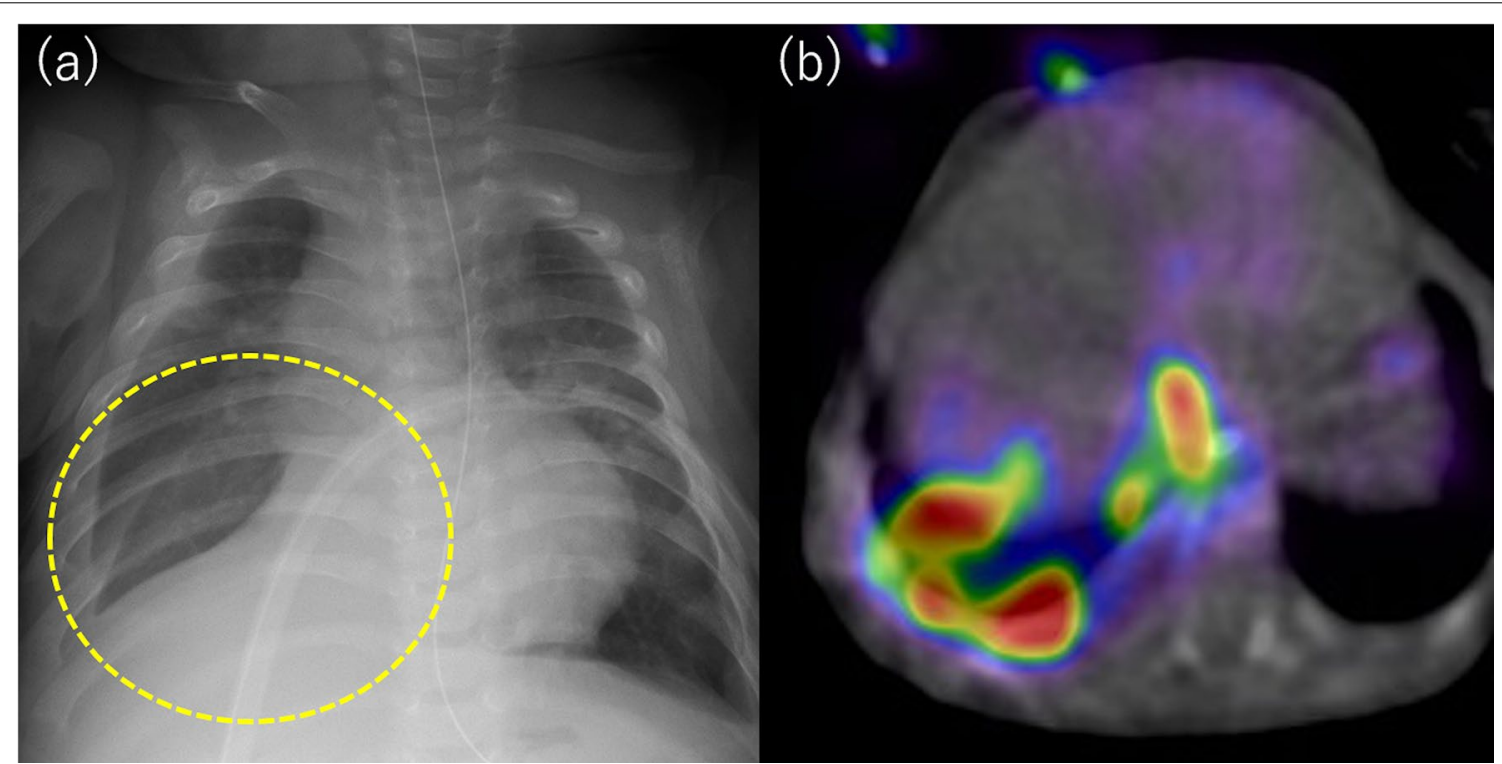

Fig. 4 a A chest radiograph showed a right-sided hydrothorax. b Preoperative scintigraphy demonstrating the radioactive dialysate in the thoracic cavity confirming a PPC 

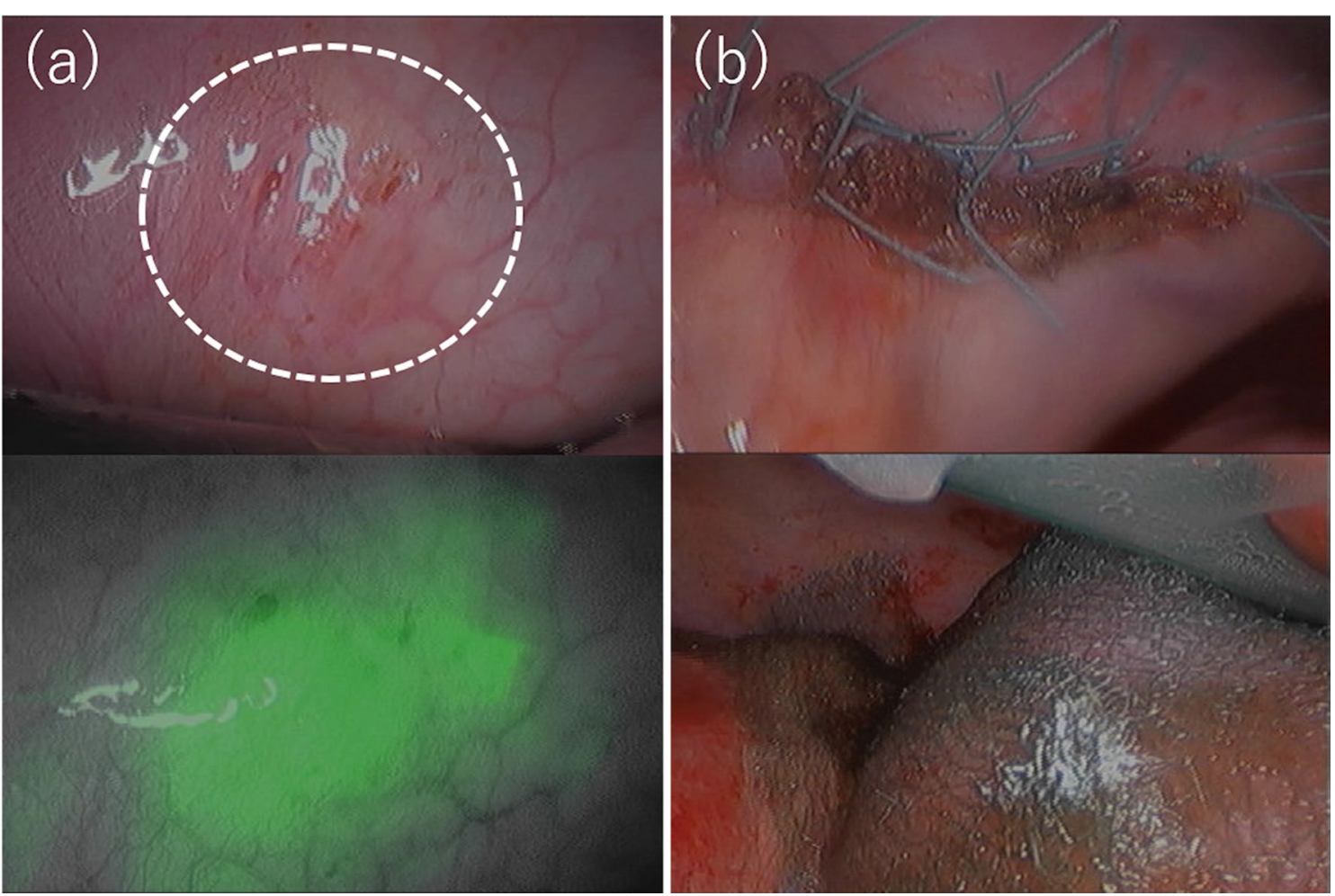

Fig. 5 Thoracoscopic findings. The pleural fluid was not stained, but an infrared camera detected fluorescence in the pleural effusion. a Small pores were detected. The fluorescence pleural effusion leaked from this site. $\mathbf{b}$ The lesion was excised and sutured directly, and a sheet of absorbable polyglycolic acid felt was fixed with fibrin glue onto the sutured lesion

\section{Discussion}

Pleural effusion due to PPC in patients undergoing PD was first described in 1967 by Edward and Ungar [8], and its incidence rate was reported 0.64 to $5.1 \%$ in adults [1, $6]$ and 0.9 to $3 \%$ in children $[1,9,10]$. At our institute, we experienced two cases of PPC among 101 PD patients in the last 17 years (approximately 2\%), which incidence rate was compatible to previous reports.

The exact mechanisms of developing PPC during PD are not well understood, but it is believed that abnormality of diaphragm and/or breakdown of lymphatic network lead to the pleural effusion [11-13]. Anatomical defects are more common in the right diaphragm, and there is a more abundant lymphatic network on the right side than on the left [1]. The lateral edge of the central tendon has abundant lymphatic stomata [14]. These factors may explain the preponderance of right-sided diaphragmatic fistula.

The definitive diagnosis of PPC can be made by the color change of pleural fluid after the injection of dialysate containing a dye such as indigo carmine or ICG into the abdominal cavity through the PD catheter, and/ or the scintigraphy $[1,4]$. If the patient is under artificial ventilation, we must be careful not to misdiagnose the existence of PPC because the resultant non-physiological pressure imbalance can cause the false-negative result for the diagnosis, as in our cases.

Treatment options include conservative management and surgical repair. Only $58 \%$ of the patients achieve long-term PD therapy without recurrence of pleural effusion [6].

Conservative treatments include spontaneous closure of the fistula by withholding PD $[1,4]$ and chemical pleurodesis [2]. In our cases, we withheld PD for 3 months, as suggested by previous reports $[15,16]$; however, this strategy was ineffective. Chemical pleurodesis therapy was not selected because of its low success rate and the possibility of inducing adhesions, which results in difficulty with the surgical approach.

Surgical repair is done by the either technique, thoracotomy or video-assisted thoracoscopic surgery. In 1984, Pattison et al. [17] reported the first case of surgical treatment using a Teflon patch for fistula closure and reported that if the fistula site could not be clearly identified, the success rate dropped to $38 \%$ and there was relatively high recurrence rate. In 1996, Di Bisceglie et al. [18] reported on the usefulness of VATS. Since then, VATS has become the standard procedure 
for the disease in adult patients, with a success rate of approximately $90 \%$, and approaching $100 \%$ when specific lesions such as cysts or flaws have been confirmed $[19,20]$.

We selected VATS technique for our patients although they were under 2-year-old infants because it allows for a thorough observation of the lesions during surgery. To identify the fistula site, a dialysate containing indigo carmine and ICG was injected into the abdominal cavity through a PD catheter. By using these assistive procedures, the lesion could be identified and treated successfully. Among the dyes, only ICG was detected in the operative field. The sensitivity of the infrared camera for fluorescence might be much higher than that of the endoscopic visible light. Thus, we recommend that ICG would be preferred as an aid during surgery.

In case 1, a laparoscope was inserted for PD catheter replacement, and a small hole was identified on the peritoneal surface of the diaphragm. Since direct suturing could tear fragile tissue and an absorbable polyglycolic acid felt could cause undesirable adhesions and reduce dialysis efficiency, autologous tissue (the falciform ligament) was used for patch closure. It may be useful to observe the abdominal cavity when the communication site cannot be identified by thoracoscopy.

\section{Conclusion}

We report two infantile cases of severe PPC associated with PD. Using endoscopic surgery and intraoperative visualization techniques (ICG navigation), we confirmed the critical communicating site and successfully closed the fistula. Even in very small infants, surgical treatment to PPC is effective, by identifying the fistula site using currently available methods.

\section{Abbreviations \\ ICG: Indocyanine green; PD: Peritoneal dialysis; PPC: Pleuroperitoneal com- munication; SPECT/CT: Single-photon emission computed tomography and computed tomography; VATS: Video-assisted thoracic surgery.}

\section{Authors' contributions}

TM is the first and the corresponding author of this manuscript. AF, MT and RF participated in the patients' care. TK, MK, AY and YK participated in the postoperative care of the patients. RS, KN and KK participated in the diagnostic procedure of the patients and participated in the perioperative care. MK read the image findings of scintigraphy. All authors critically revised the report, commented on drafts of the manuscript. All authors read and approved the final manuscript.

\section{Funding}

This study was supported by a Grant from the Research Project for Intractable Diseases by the Ministry of Health, Labour and Welfare in Japan (Grant no. JPMH2OFC1017) received by the NU.

\section{Availability of data and materials}

All related data are included with the article.

\section{Declarations}

Ethics approval and consent to participate

Ethical approval was obtained from the ethics committee of our institute.

Consent for publication

Informed consent for publication was obtained from the parents of the patients.

\section{Competing interests}

The authors declare that they have no known competing financial interests or personal relationships that could have influenced the work reported in this manuscript.

\section{Author details}

${ }^{1}$ Division of Pediatric Surgery, National Center for Child Health and Development, 2-10-1 Okura Setagaya-ku, Tokyo 157-8535, Japan. ${ }^{2}$ Division of Nephrology and Rheumatology, National Center for Child Health and Development, Tokyo, Japan. ${ }^{3}$ Division of Radiology, National Center for Child Health and Development, Tokyo, Japan.

Received: 7 June 2021 Accepted: 5 Auqust 2021

Published online: 12 August 2021

\section{References}

1. Nomoto Y, Suga T, Nakajima K, et al. Acute hydrothorax in continuous ambulatory peritoneal dialysis-a collaborative study of 161 centers. Am J Nephrol. 1989;9:363-7.

2. Aksoy OY, Gulaldi NCM, Bayrakci US. Hydrothorax in a pediatric patient on peritoneal dialysis: answers. Pediatr Nephrol. 2020;35:1421-3.

3. Yaxley J, Twomey K. Peritoneal dialysis complicated by pleuroperitoneal communication and hydrothorax. Ochsner J. 2017;17:124-7.

4. Tang S, Chui WH, Tang AW, et al. Video-assisted thoracoscopic talc pleurodesis is effective for maintenance of peritoneal dialysis in acute hydrothorax complicating peritoneal dialysis. Nephrol Dial Transplant. 2003;18:804-8.

5. Matsumura M, Mizutani E, Suzuki T, et al. Successful diagnosis and treatment of peritoneal dialysis-related pleuroperitoneal communication by contrast-enhanced ultrasonography and thoracoscopic surgery. Perit Dial Int. 2017;37:485-6.

6. Chow KM, Szeto CC, Li PK. Management options for hydrothorax complicating peritoneal dialysis. Semin Dial. 2003;16:389-94.

7. Dufek S, Holtta T, Fischbach M, et al. Pleuro-peritoneal or pericardioperitoneal leak in children on chronic peritoneal dialysis - a survey from the European Paediatric Dialysis Working Group. Pediatr Nephrol. 2015;30:2021-7.

8. Edwards SR, Unger AM. Acute hydrothorax - a new complication of peritoneal dialysis. JAMA. 1967;199:853-5.

9. Kawaguchi AL, Dunn JC, Fonkalsrud EW. Management of peritoneal dialysis-induced hydrothorax in children. Am Surg. 1996;62:820-4

10. Verrina E, Bassi S, Perfumo F, et al. Analysis of complications in a chronic peritoneal dialysis pediatric patient population. The Italian Registry of Pediatric Chronic Peritoneal Dialysis. Perit Dial Int. 1993;13(Suppl 2):S257-9.

11. Gagnon RF, Daniels E. The persisting pneumatoenteric recess and the infracardiac bursa: possible role in the pathogenesis of right hydrothorax complicating peritoneal dialysis. Adv Perit Dial. 2004;20:132-6.

12. Lew SQ. Hydrothorax: pleural effusion associated with peritoneal dialysis. Perit Dial Int. 2010;30:13-8.

13. Johnston RF, Loo RV. Hepatic hydrothorax; studies to determine the source of the fluid and report of thirteen cases. Ann Intern Med. 1964;61:385-401.

14. Nakatani T, Ohtani O, Tanaka S. Lymphatic stomata in the murine diaphragmatic peritoneum: the timing of their appearance and a map of their distribution. Anat Rec. 1996;244:529-39.

15. Gidaris D, Printza N, Batzios S, Belechri AM, Papachristou F. A "sweet" hydrothorax in a child on peritoneal dialysis. Hippokratia. 2011;15:358-60. 
16. Leblanc M, Ouimet D, Pichette V. Dialysate leaks in peritoneal dialysis. Semin Dial. 2001;14:50-4.

17. Pattison CW, Rodger RS, Adu D, Michael J, Matthews HR. Surgical treatment of hydrothorax complicating continuous ambulatory peritoneal dialysis. Clin Nephrol. 1984;21:191-3.

18. Di Bisceglie M, Paladini P, Voltolini L, et al. Videothoracoscopic obliteration of pleuroperitoneal fistula in continuous peritoneal dialysis. Ann Thorac Surg. 1996;62:1509-10.

19. Mitsuboshi S, Maeda H, Kanzaki M. Video-assisted thoracic surgery for pleuroperitoneal communication. Surg Case Rep. 2019;5:34.
20. Manabe T, Ono K, Oka S, Kawamura Y, Osaki T. A case of pleuroperitoneal communication in which establishing a laparoscopic pneumoperitoneum was useful for the detection of a fistula. Surg Case Rep. 2021;7:64

\section{Publisher's Note}

Springer Nature remains neutral with regard to jurisdictional claims in published maps and institutional affiliations.

\section{Submit your manuscript to a SpringerOpen ${ }^{\circ}$ journal and benefit from:}

- Convenient online submission

- Rigorous peer review

- Open access: articles freely available online

- High visibility within the field

- Retaining the copyright to your article

Submit your next manuscript at $\boldsymbol{\nabla}$ springeropen.com 